\title{
Editorial
}

\section{Nonlinear and Noncommutative Mathematics: New Developments and Applications in Quantum Physics}

\author{
M. Norbert Hounkonnou, ${ }^{1}$ S. Twareque Ali, ${ }^{2}$ Gerald A. Goldin, ${ }^{3}$ \\ Richard Kerner, ${ }^{4}$ Kalyan B. Sinha, ${ }^{5}$ and Akira Yoshioka ${ }^{6}$ \\ ${ }^{1}$ International Chair in Mathematical Physics and Applications (ICMPA-UNESCO Chair), \\ University of Abomey-Calavi (UAC), 072 BP 50, Cotonou, Benin \\ ${ }^{2}$ Department of Mathematics and Statistics, Concordia University, 1455 de Maisonneuve Blvd. West, \\ Montreal, Quebec, Canada H3G 1M8 \\ ${ }^{3}$ Departments of Mathematics and Physics, Rutgers University, Piscataway, NJ 08854, USA \\ ${ }^{4}$ Laboratoire de Physique Théorique de la Matière Condensée (LPTMC), Université Pierre et Marie Curie, \\ 4 Place Jussieu, 75005 Paris, France \\ ${ }^{5}$ Jawaharlal Nehru Centre for Advanced Scientific Research, Indian Institute of Science, Bangalore, India \\ ${ }^{6}$ Department of Mathemtaics, Faculty of Science, Tokyo University of Science, Kagurazaka 1-3, \\ Shinjyuku-ku, Tokyo 162-8601, Japan \\ Correspondence should be addressed to M. Norbert Hounkonnou, \\ norbert.hounkonnou@cipma.uac.bj \\ Received 8 September 2010; Accepted 8 September 2010
}

Copyright (C 2010 M. Norbert Hounkonnou et al. This is an open access article distributed under the Creative Commons Attribution License, which permits unrestricted use, distribution, and reproduction in any medium, provided the original work is properly cited.

\section{Introduction to the Special Issue}

This Special Issue of Advances in Mathematical Physics addresses a number of contemporary topics, centered in the domains (broadly construed) of nonlinear and noncommutative mathematics. These encompass some rapidly developing, versatile areas without, of course, well-defined boundaries. They not only suggest mathematical generalizations in many different directions, but their applications in theoretical and mathematical physics are diverse-in the fields of quantization, quantum theory, and quantum information, in highenergy physics and quantum gravity, in plasma physics and condensed matter theory, and in other areas.

The ten articles in the Special Issue can address but a small subset of these fields, making use of a cross-section of mathematical methods. Some of the papers are intended to present reviews of important areas, while others are more specifically focused. In the latter case, we nevertheless sought to encourage the authors to provide some general background. 


\section{Quantization and Quantum Field Theory}

The issue begins with a set of four articles directly pertinent to problems of quantization and quantum field theory, while the methods described also have many other important applications. The review by Martin Schlichenmaier provides a succinct yet detailed exposition of the key concepts in Berezin-Toeplitz and the related deformation quantization for compact Kähler manifolds. This method of quantization has been theoretically one of the most useful quantization techniques, the strongest results in the field coming from compact manifolds. The author also points out possible uses of this technique in the study of noncommutative geometry, for example, fuzzy spheres. Next Jean-Pierre Antoine and Camillo Trapani present an overview of partial inner product spaces and operators on them, with the results illustrated by families of function spaces that are important in signal analysis as well as mathematical physics. While the Hilbert space as the state space is germane to quantum mechanics, the use of operators with continuous spectra is traditionally handled within the Dirac bra-ket scheme which, mathematically speaking, involves the use of Gelfand triples. A mathematically rigorous and adequate framework for such a structure is the more general concept of a partial inner product space. Similarly, in signal processing, where continuous scales of smoothness are often required, the use of partial inner product spaces becomes particularly useful.

In the third article, John Klauder defines and analyzes coherent states associated with covariant, non-renormalizable, self-interacting scalar fields quantized so as to satisfy affine rather than canonical commutation relations. Coherent states are well known objects in physics and in analysis. The present article analyzes affine coherent states, built using the position and dilatation operators, starting from the ground state of a lattice version of a covariant, non-renormalizable, self-interacting, scalar quantum field. Then Ivan Todorov reviews his joint work with B. Bakalov, N. M. Nikolov and K.-H. Rehren on gauge symmetry and Howe duality in a local scalar field theory of conformal dimension two in fourdimensional space-time. The review extends the results obtained in the two-dimensional conformal field theories to four dimensions. Possible models are surveyed and the infinitedimensional Lie algebras that naturally arise in this context are investigated. One of the most important results is the example of realization of the Doplicher-Haag-Roberts' theory of superselection sectors and compact gauge groups. This survey article has great pedagogical value and will become one of the best sources for all those who are interested in conformal field theories.

\section{Two-Dimensional Projective Geomeotry}

Following these extensive reviews, we include a short mathematical article by Kyousuke Uchino using lattice theory to explain V. Arnold's two-dimensional projective geometry. Arnold considered the set of all homogeneous quadratic forms of two variables, which is a Poisson algebra by the canonical Poisson bracket. The point of Arnold's idea is that the points of the projective plane are replaced with quadratic forms. Using the canonical Poisson bracket, Arnold showed that the Jacobi identity of the Poisson algebra gives the altitude theorem. Extending Arnold's idea Tomihisa gave proofs of fundamental theorems of projective planes, such as Pappus' theorem, Pascal's theorem and Brianchon's theorem by means of the Poisson algebra. Uchino gives a clear view of these arguments by means of the lattice structure of a Lie algebra. Using the Plucker embedding, Uchino shows that the two-dimensional projective geometry is encoded in $s l(2)$. As an application, the classical 
Yang-Baxter equation is translated into the geometry of a quadratic curve on the projective plane.

\section{Infinite-Dimensional Groups and Algebras, Gauge Theories, Topological String Theory, and Instantons}

The next paper in this special issue is a wide-ranging survey by Rudolf Schmid of infinitedimensional Lie groups and algebras, including diffeomorphism groups, gauge groups, and loop groups, together with some of their areas of application in mathematical physics. Mathematically, infinite-dimensional Lie groups, being non-locally compact, do not satisfy many of the elegant properties of finite dimensional groups. Physically, however, they can describe the symmetries of systems (including nonlinear systems) having infinitelymany degrees of freedom; and they can encode mathematically the important idea of local symmetry. The areas of application discussed in this article range from electromagnetism (Maxwell's equations), hydrodynamics (Euler's equations), and plasma physics (MaxwellVlasov equations) to the Korteweg-de Vries equation, BRST symmetry, the standard model, gravity, and supersymmetry.

We then present a review by Richard Szabo of some results in gauge theories and topological string theory. Connections are developed between instanton counting in maximally supersymmetric gauge theories, and invariants of smooth varieties. The counting problems discussed by Szabo connect six-dimensional topological string theory/gauge theory, four-dimensional supersymmetric gauge theories, three-dimensional Chern Simons theory, and two-dimensional q-deformed Yang-Mills theory, as well as two-dimensional conformal field theory-with relationships to the entropy of supersymmetric black holes and, for example, Donaldson-Thomas and Gromov-Witten invariants. These topological models, obtained as topological twists of a given physical theory, can serve as exactly solvable systems which capture the physical content of certain sectors of more elaborate systems with local propagating degrees of freedom. They describe the BPS sectors of physical models, and compute nonperturbative effects therein. Thus, for certain supersymmetric charged black holes, the microscopic Bekenstein-Hawking-Wald entropy is computed by the Witten index of the relevant supersymmetric gauge theory. This is equivalent to the counting of stable BPS bound states of D-branes in the pertinent geometry, and is related to invariants of three-folds via the OSV conjecture.

There follows Akifumi Sako's review of four-dimensional noncommutative instantons, focusing on some recent perspectives and showing, for example, how the noncommutative instanton charges coincide with the charges of the instanton solutions prior to noncommutative deformation.

\section{Additional Topics and Innovative Methods}

The final two articles in this Special Issue again present more specific results. Rémi Léandre discusses white noise analysis and quantum probability (described by means of a bosonic Fock space), and Malliavin Calculus (for the Wiener measure in $L^{\mathrm{p}}$ space), constructing a Poisson structure and a Lie algebroid on the space of smooth 1-forms on the Wiener space using the Nualart-Pardoux definition of Anticipative Stratanovich integrals. The development, enriched by a good list of relevant biographical references, contains interesting comments on the implications of relevant results and clarifies various concepts in the field. 
Finally, A. Bostan et al. draw our attention to a variety of essential concepts as they exemplify exact representations of the renormalization group as isogenies of elliptic curves using linear differential equations covariant under commuting rational transformations of infinite order. These novel and original results are at the crossroads of many domains of mathematical physics, like the theory of elliptic curves and modular forms, hypergeometric function theory and differential algebra, to name just a few. The concepts and ideas introduced in this seminal paper provide new and powerful tools for deeper investigation and analysis of lattice models of statistical mechanics, creating an unexpected bridge to Calabi-Yau manifolds and string theory. Last but not least, this paper provides an example of a fruitful collaboration, in which the skills and deep knowledge of applied mathematics, fundamental mathematics and theoretical physics brought in by co-authors coming from different countries and continents merge in a very harmonious way to give a beautiful and useful result.

Many of the papers also include substantial bibliographic resources. We expect these expositions to be a rich resource, of interest both to mathematicians and to theoretical physicists.

M. Norbert Hounkonnou S. Twareque Ali Gerald A. Goldin Richard Kerner Kalyan B. Sinha Akira Yoshioka 


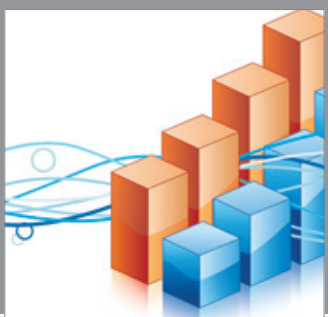

Advances in

Operations Research

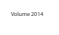

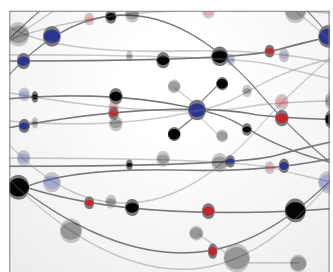

\section{The Scientific} World Journal
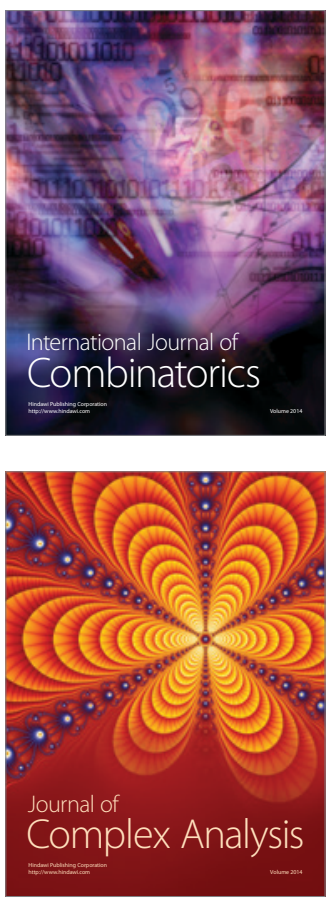

International Journal of

Mathematics and

Mathematical

Sciences
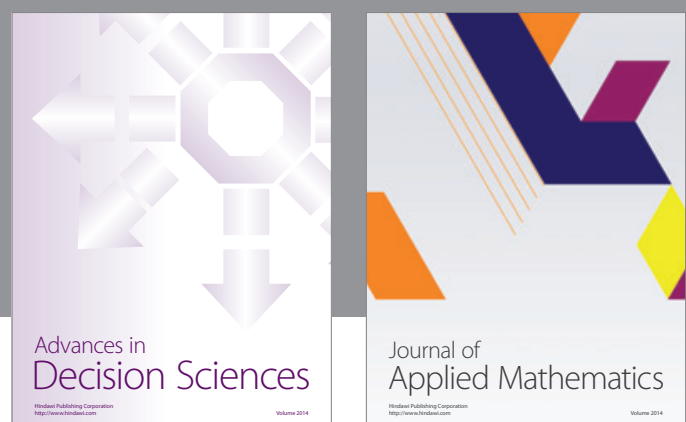

Journal of

Applied Mathematics
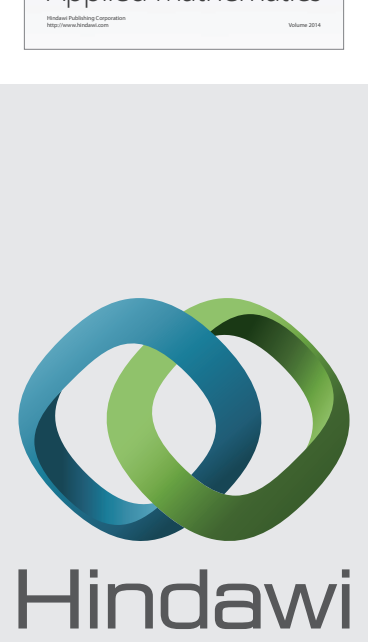

Submit your manuscripts at http://www.hindawi.com
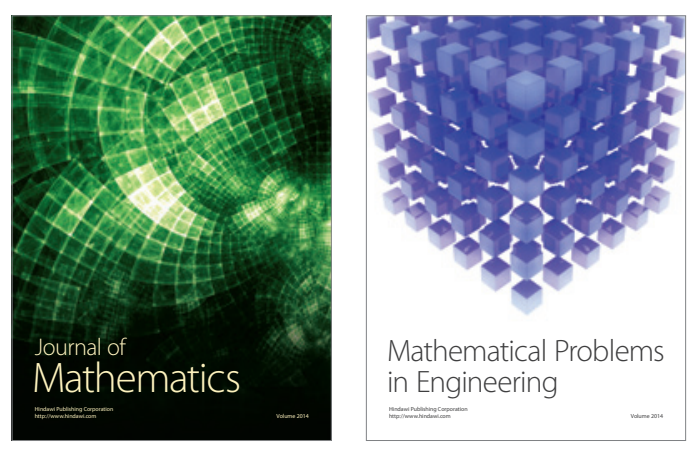

Mathematical Problems in Engineering
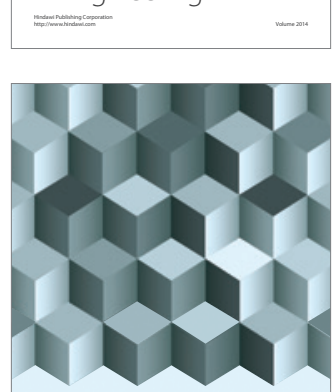

Journal of

Function Spaces
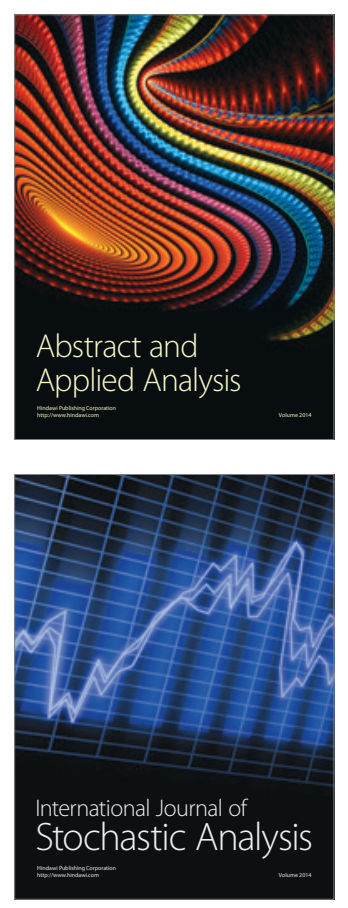

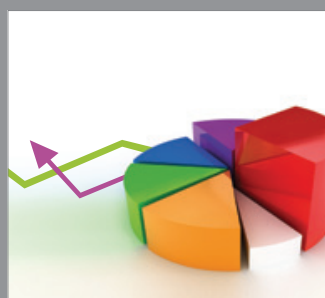

ournal of

Probability and Statistics

Promensencen
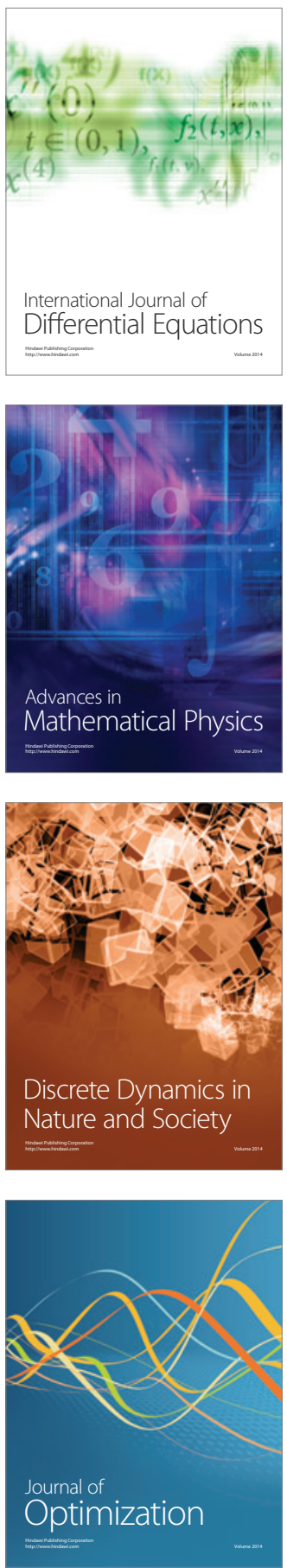